\title{
MEAN ERGODIC THEOREMS FOR NONLINEAR OPERATORS
}

\author{
RAINER WITTMANN
}

(Communicated by William J. Davis)

\begin{abstract}
The mean ergodic theorem is shown for nonlinear operators $T$ : $K \longrightarrow K$ with $\|T x+T y\| \leq\|x+y\|$ for any $x, y \in K$ where $K$ may be an arbitrary subset of a Hilbert space $H$.
\end{abstract}

\section{INTRODUCTION}

Let $K$ be a subset of Banach space $H$ and $T: K \longrightarrow K$ be a mapping. We are concerned with the mean ergodic theorem for such nonlinear operators, i.e. the norm convergence of

$$
A_{n}(x):=\frac{1}{n+1} \sum_{i=0}^{n} T^{i} x \quad(x \in K) .
$$

Such results are very well known for linear contractions. In order to generalize such results to nonlinear mappings the most natural constraint on $T$ is to assume that

$$
\|T x-T y\| \leq\|x-y\| \quad(x, y \in K) .
$$

Such mappings are called nonexpansive. Unfortunately there exist examples of nonexpansive operators where $K=H$ is a Hilbert space and $T$ is nonexpansive with nonempty fixed point set but the mean ergodic theorem does not hold (although $A_{n} x$ converges weakly). The speed limit operators of Krengel and Lin [6] are such examples if the speed function is chosen appropriately. Moreover, these operators satisfy several additional natural conditions. On the other hand Baillon [1] was able to prove the mean ergodic theorem for nonexpansive operators $T$ on a convex subset $K$ of a Hilbert space if $-x \in K$ and $T(-x)=-T x$ for any $x \in K$. Clearly the last condition and (1) imply

$$
\|T x+T y\| \leq\|x+y\| \quad(x, y \in K) .
$$

Received by the editors April 9, 1989 and, in revised form, July 3, 1989.

1980 Mathematics Subject Classification (1985 Revision). Primary 46B30, 47A35.

Key words and phrases. Mean ergodic theorem, Fixed point theorem, Nonlinear operators, Hilbert spaces.

The author is a Heisenberg Fellow of Deutsche Forschungsgemeinschaft. 
In this paper we show that (2) alone guarantees the validity of the mean ergodic theorem if $H$ is a Hilbert space. Note that (2) does not even imply continuity. On the other hand, if $-x \in K$ for any $x \in K$, then (2) implies (1). In fact, $\|T x+T(-x)\| \leq\|x+(-x)\|=0$, implies $T(-x)=-T x$ and therefore $\|T x-T y\|=\|T(-x)+T y\| \leq\|x-y\|$. But also in this case our result is better than that of Baillon because we need not impose any convexity assumption on $K$.

Hirano and Takahashi [4] have replaced (1) by the weaker condition

$$
\begin{aligned}
& \left\|T^{n} x-T^{n} y\right\| \leq \alpha_{n}\|x-y\| \quad(x, y \in K), \\
& \lim _{n \rightarrow \infty} \alpha_{n}=1,
\end{aligned}
$$

and proved some weak convergence results for $A_{n} x$. If $-x \in K$ and $T(-x)=$ $-T x$ then $\left(1^{\prime}\right)$ implies

$$
\begin{aligned}
& \left\|T^{n} x+T^{n} y\right\| \leq \alpha_{n}\|x+y\| \quad(x, y \in K), \\
& \lim _{n \rightarrow \infty} \alpha_{n}=1 .
\end{aligned}
$$

We will show that the mean ergodic theorem still holds if only $\left(2^{\prime}\right)$ is satisfied.

Even the results about weak convergence of $A_{n} x$ for nonexpansive mappings imply that the limit is a fixed point. As Example 3.2 shows this need no more hold under our assumptions even if $T$ is continuous. Unlike the case of nonexpansive operators the fixed point set need no more be convex, but it is always nonempty if $K$ is a convex and closed set. In this case the unique point $x_{0} \in K$ with $\left\|x_{0}\right\|=\inf \{\|x\|: x \in K\}$ is always a fixed point because $\left\|T x_{0}\right\| \leq\left\|x_{0}\right\|$ by (2).

For further information on nonlinear ergodic theorems the reader is referred to Bruck [2], $\S 9.3$ of Krengel [5] and the literature cited there.

After having finished a first draft of this paper Professors U. Krengel and M. Lin drew the author's attention to the unpublished preprint [3] of DjafariRouhani and Kakutani. There, the particular case $\delta_{k}=0$ of our main result Theorem 2.3 is stated, with the additional assumption $\left\|X_{n+1}-X_{m+1}\right\| \leq$ $\left\|X_{n}-X_{m}\right\|$ for any $n, m \in \mathbf{N}$, but since the proof was incomplete, [3] was never published (although it contains another important result which was later generalized in [6]).

\section{MAIN STREAM}

In the sequel $H$ will always be a Hilbert space with inner product $\langle\cdot, \cdot\rangle$ and norm $\|\cdot\|$.

Theorem 2.1. Let $K \subset H$ and $T: K \longrightarrow K$ be a mapping satisfying

$$
\|T x+T y\| \leq\|x+y\| \quad(x, y \in K) .
$$

Then for any $x \in K$

$$
A_{n} x:=\frac{1}{n+1} \sum_{i=0}^{n} T^{i} x
$$

is norm convergent. 
Theorem 2.1 is a special case of

Theorem 2.2. Let $K \subset H$ and $T: K \longrightarrow K$ be a mapping satisfying

(i)

$$
\begin{aligned}
& \left\|T^{n} x+T^{n} y\right\| \leq \alpha_{n}\|x+y\| \quad(x, y \in K), \\
& \lim _{n \rightarrow \infty} \alpha_{n}=1 .
\end{aligned}
$$

Then for any $x \in K$

$$
A_{n} x:=\frac{1}{n+1} \sum_{i=0}^{n} T^{i} x
$$

is norm convergent.

Theorem 2.2 is an immediate consequence of

Theorem 2.3. Let $\left(X_{n}\right)$ be a sequence in $H$ such that

$$
\left\|X_{n+k}+X_{m+k}\right\|^{2} \leq\left\|X_{n}+X_{m}\right\|^{2}+\delta_{k} \quad(m, n, k \in \mathbf{N})
$$

with

$$
\lim _{k \rightarrow \infty} \delta_{k}=0
$$

Then the sequence $A_{n}$ defined by

$$
A_{n}:=\frac{1}{n} \sum_{i=1}^{n} X_{i}
$$

is norm convergent.

Remark. Clearly Theorem 2.3 contains also the mean ergodic theorem for stationary processes in the wide sense (cf. [5], p. 32).

Proof. From (i) and (ii) we see that for any $m, n \in N$

$$
\limsup _{k \rightarrow \infty}\left\|X_{n+k}+X_{m+k}\right\|=\liminf _{k \rightarrow \infty}\left\|X_{n+k}+X_{m+k}\right\|=\inf _{k \in N}\left\|X_{n}+X_{m}\right\|
$$

Taking $m=n=1$ this implies

$$
n_{\varepsilon}:=\inf \left\{n \in \mathbf{N}:\left\|X_{m_{1}}\right\|^{2} \leq\left\|X_{m_{2}}\right\|^{2}+\varepsilon / 2 \quad \forall m_{2} \geq m_{1} \geq n_{\varepsilon}\right\}<\infty .
$$

For any $\varepsilon>0$ we set

$$
k_{\varepsilon}:=\inf \left\{k \in \mathbf{N}: \delta_{n} \leq \varepsilon \quad \forall n \geq k\right\} .
$$

Since

$$
\left\langle X_{n}, X_{m}\right\rangle=\frac{1}{2}\left(\left\|X_{n}+X_{m}\right\|^{2}-\left\|X_{n}\right\|^{2}-\left\|X_{m}\right\|^{2}\right)
$$

we obtain for any $m, n \geq n_{\varepsilon}$ and $k \geq k_{\varepsilon}$

$$
\begin{aligned}
\left\langle X_{n+k}, X_{m+k}\right\rangle & =\frac{1}{2}\left(\left\|X_{n+k}+X_{m+k}\right\|^{2}-\left\|X_{n+k}\right\|^{2}-\left\|X_{m+k}\right\|^{2}\right) \\
& \leq \frac{1}{2}\left(\left\|X_{n}+X_{m}\right\|^{2}+\varepsilon-\left\|X_{n}\right\|^{2}+\frac{\varepsilon}{2}-\left\|X_{m}\right\|^{2}+\frac{\varepsilon}{2}\right) \\
& =\left\langle X_{n}, X_{m}\right\rangle+\varepsilon .
\end{aligned}
$$


For any $\varepsilon>0$ we define

$$
\begin{aligned}
& K_{\varepsilon}:=\left\{\sum_{i=0}^{p} t_{i} X_{n_{\varepsilon}+i}: p \in \mathbf{N}, \quad 0 \leq t_{i} \leq 1, \quad \sum_{i=0}^{p} t_{i}=1\right\}, \\
& I_{\varepsilon}=\inf _{z \in K_{\varepsilon}}\|z\|^{2}
\end{aligned}
$$

and choose $p_{\varepsilon} \in \mathbf{N},\left(t_{\varepsilon, i}\right)_{0 \leq i \leq p_{\varepsilon}}$ in $[0,1]$ such that $\sum_{i=0}^{p_{\varepsilon}} t_{\varepsilon, i}=1$ and

$$
\left\|\sum_{i=0}^{p_{\varepsilon}} t_{\varepsilon, i} X_{n_{\varepsilon}+i}\right\|^{2} \leq I_{\varepsilon}+\varepsilon .
$$

Using (1) we obtain for any $\varepsilon>0$ and $k \geq k_{\varepsilon}$

$$
\begin{aligned}
\left\|\sum_{i=0}^{p_{\varepsilon}} t_{\varepsilon, i} X_{n_{\varepsilon}+k+i}\right\|^{2} & =\sum_{i, j=0}^{p_{\varepsilon}} t_{\varepsilon, i} t_{\varepsilon, j}\left\langle X_{n_{\varepsilon}+k+i}, X_{n_{\varepsilon}+k+j}\right\rangle \\
& \leq \sum_{i, j=0}^{p_{\varepsilon}} t_{\varepsilon, i} t_{\varepsilon, j}\left\langle X_{n_{\varepsilon}+i}, X_{n_{\varepsilon}+j}\right\rangle+\sum_{i, j=0}^{p_{\varepsilon}} t_{\varepsilon, i} t_{\varepsilon, j} \varepsilon \\
& =\left\|\sum_{i=0}^{p_{\varepsilon}} t_{\varepsilon, i} X_{n_{\varepsilon}+i}\right\|^{2}+\varepsilon .
\end{aligned}
$$

Together with the convexity of $x \longrightarrow x^{2}$ and (2) this implies

$$
\left\|z_{\varepsilon, m}\right\|^{2} \leq I_{\varepsilon}+\varepsilon \quad(m \in \mathbf{N}, \varepsilon>0)
$$

where

$$
z_{\varepsilon, m}:=\frac{1}{m+1} \sum_{k=k_{\varepsilon}}^{k_{\varepsilon}+m} \sum_{i=0}^{p_{\varepsilon}} t_{\varepsilon, i} X_{n_{\varepsilon}+k+i} .
$$

Note that $\varepsilon \longrightarrow I_{\varepsilon}$ is decreasing. Hence

$$
I_{0}:=\lim _{\varepsilon \rightarrow 0} I_{\varepsilon}
$$

exists and for any $\alpha>0$ we can define

$$
\varepsilon_{\alpha}:=\sup \left\{\varepsilon>0: I_{\varepsilon} \geq I_{0}-\alpha\right\} .
$$

Now let $\alpha>0$ and $0<\delta<\varepsilon<\varepsilon_{\alpha}$ be given. Since $\frac{1}{2}\left(z_{\varepsilon, m}+z_{\delta, n}\right) \in K_{\varepsilon}$ we have

$$
\left\|\frac{1}{2}\left(z_{\varepsilon, m}+z_{\delta, n}\right)\right\|^{2} \geq I_{\varepsilon} \geq \frac{1}{2}\left(I_{\varepsilon}+I_{0}-\alpha\right) \geq \frac{1}{2}\left(I_{\varepsilon}+I_{\delta}-\alpha\right) .
$$

Combining with (3) we obtain

$2\left\|z_{\varepsilon, m}\right\|^{2}+2\left\|z_{\delta, n}\right\|^{2}-\left\|z_{\varepsilon, m}+z_{\delta, n}\right\|^{2} \leq 2 I_{\varepsilon}+2 \varepsilon+2 I_{\delta}+2 \delta-2 I_{\varepsilon}-2 I_{\delta}+2 \alpha \leq 4 \varepsilon+2 \alpha$.

By the parallelogram identity the left-hand side of the last inequality equals $\left\|z_{\varepsilon, m}-z_{\delta, n}\right\|^{2}$. Thus we have shown

$$
\left\|z_{\varepsilon, m}-z_{\delta, n}\right\|^{2} \leq 4 \varepsilon+2 \alpha \quad\left(0<\delta<\varepsilon<\varepsilon_{\alpha}, m, n \in \mathbf{N}\right) .
$$


Since $H$ is a complete space there exists $z \in H$ with

$$
\left\|z_{\varepsilon, m}-z\right\|^{2} \leq 4 \varepsilon+2 \alpha \quad\left(0<\varepsilon<\varepsilon_{\alpha}, m \in \mathbf{N}\right) .
$$

From the definition of $z_{\varepsilon, m}$ it is easy to see that

$$
(m+1) z_{\varepsilon, m}-\sum_{i=n_{\varepsilon}+p_{\varepsilon}+k_{\varepsilon}}^{n_{\varepsilon}+k_{\varepsilon}+m} X_{i}=\sum_{i=0}^{p_{\varepsilon}-1} r_{i} X_{n_{\varepsilon}+k_{\varepsilon}+i}+\sum_{i=1}^{p_{\varepsilon}} s_{i} X_{n_{\varepsilon}+k_{\varepsilon}+m+i}
$$

with $0 \leq r_{i}, s_{i} \leq 1$ if $m \geq p_{\varepsilon}$. Hence

$$
\left\|z_{\varepsilon, m}-A_{m+1}\right\| \leq \frac{1}{m+1}\left(2 p_{\varepsilon} \sup _{n \in \mathbf{N}}\left\|X_{n}\right\|+\left(n_{\varepsilon}+k_{\varepsilon}\right) \sup _{n \in \mathbf{N}}\left\|X_{n}\right\|\right) .
$$

Letting $m$ tend to infinity (4) now yields

$\limsup _{n \rightarrow \infty}\left\|A_{m}-z\right\|^{2} \leq 2 \sup _{m \in \mathbf{N}}\left\|z_{\varepsilon, m}-z\right\|^{2}+2 \limsup _{n \rightarrow \infty}\left\|A_{m+1}-z_{\varepsilon, m}\right\|^{2} \leq 8 \varepsilon+4 \alpha+0$.

Since $\alpha>0$ and $\varepsilon>0$ can be made arbitrarily small the assertion follows.

\section{EXAMPLES}

The first two examples below are quite pathological. Their main purpose is to show the difference to the case of nonexpansive operators.

Example 3.1. Let $\mu$ be a $\sigma$-finite measure and $L_{+}^{2}$ be the convex cone of all positive functions of the Hilbert space $L^{2}(\mu)$. Further let $\phi: L_{+}^{2} \longrightarrow[0,1]$ be an arbitrary function. Then the mapping $T_{\phi} f:=\phi(f) f$ clearly satisfies

$$
\left\|T_{\phi} f+T_{\phi} g\right\| \leq\|f+g\| \quad\left(f, g \in L_{+}^{2}\right) .
$$

On the other hand, even when $L_{+}^{2}$ is one dimensional, i.e. $\mu$ is a point mass, one can easily give examples of functions $\phi$ such that the fixed point set is not convex and $T_{\phi}$ is not continuous. Moreover, $\phi$ may be chosen such that $T_{\phi}$ is order preserving on $L_{+}^{2}$.

Even if $T$ seems to be nice the ergodic averages may behave pathologically :

Example 3.2. We define

$$
T\left(x_{1}, x_{2}\right)= \begin{cases}\frac{\max \left(x_{1}^{2}, x_{2}^{2}\right)}{x_{1}^{2}+x_{2}^{2}}\left(x_{2}, x_{1}\right) & \text { if }\left(x_{1}, x_{2}\right) \in \mathbf{R}_{+}^{2} \backslash\{(0,0)\}, \\ 0 & \text { if }\left(x_{1}, x_{2}\right)=(0,0) .\end{cases}
$$

Again we have

$$
\left\|T\left(x_{1}, x_{2}\right)+T\left(y_{1}, y_{2}\right)\right\| \leq\left\|\left(x_{1}, x_{2}\right)+\left(y_{1}, y_{2}\right)\right\| \quad\left(\left(x_{1}, x_{2}\right),\left(y_{1}, y_{2}\right) \in \mathbf{R}_{+}^{2}\right)
$$

and $T$ is continuous on $\mathbf{R}_{+}^{2}$ with $(0,0)$ as the only fixed point.

On the other hand, if $x>0$ the averages

$$
\frac{1}{n+1} \sum_{i=0}^{n} T^{i}(x, 0)
$$

converge to $\left(\frac{1}{2} x, \frac{1}{2} x\right)$ which is not a fixed point. 
We now turn over to more natural examples. In the sequel $\mu$ will be a $\sigma$-finite measure. The following lemma is basic:

Lemma 3.3. Let $X, Y, X^{\prime}, Y^{\prime} \in L^{4}(\mu)$ and $\delta \geq 0$ such that

(i) $\|X\|_{4}^{4} \leq\left\|X^{\prime}\right\|_{4}^{4}+\delta / 2$,

(ii) $\|Y\|_{4}^{4} \leq\left\|Y^{\prime}\right\|_{4}^{4}+\delta / 2$,

(iii) $\|X+Y\|_{4}^{4} \leq\left\|X^{\prime}+Y^{\prime}\right\|_{4}^{4}+\delta$,

(iv) $\|X-Y\|_{4}^{4} \leq\left\|X^{\prime}-Y^{\prime}\right\|_{4}^{4}+\delta$.

Then we have

$$
\left\|X^{2}+Y^{2}\right\|_{2}^{2} \leq\left\|X^{\prime 2}+Y^{\prime 2}\right\|_{2}^{2}+\delta .
$$

Proof. For simplicity we use the probabilistic notation

$$
E(Z)=\int Z d \mu
$$

From (iii) and (iv) we get

$$
\begin{aligned}
2 E\left(X^{4}\right)+2 E\left(Y^{4}\right)+12 E\left(X^{2} Y^{2}\right) & =E\left((X+Y)^{4}\right)+E\left((X-Y)^{4}\right) \\
& \leq E\left(\left(X^{\prime}+Y^{\prime}\right)^{4}\right)+E\left(\left(X^{\prime}-Y^{\prime}\right)^{4}\right)+2 \delta \\
& =2 E\left(X^{\prime 4}\right)+2 E\left(Y^{\prime 4}\right)+12 E\left(X^{\prime 2} Y^{\prime 2}\right)+2 \delta
\end{aligned}
$$

and therefore

$$
\begin{aligned}
\left\|X^{2}+Y^{2}\right\|_{2}^{2}= & E\left(X^{4}\right)+E\left(Y^{4}\right)+2 E\left(X^{2} Y^{2}\right) \\
= & \frac{2}{3}\left(E\left(X^{4}\right)+E\left(Y^{4}\right)\right)+\frac{1}{6}\left(2 E\left(X^{4}\right)+2 E\left(Y^{4}\right)+12 E\left(X^{2} Y^{2}\right)\right) \\
\leq & \frac{2}{3}\left(E\left(X^{\prime 4}\right)+\delta / 2+E\left(Y^{\prime 4}\right)+\delta / 2\right)+\frac{1}{6}\left(2 E\left(X^{\prime 4}\right)+2 E\left(Y^{\prime 4}\right)\right. \\
& \left.+12 E\left(X^{\prime 2} Y^{\prime 2}\right)+2 \delta\right) \\
= & E\left(X^{\prime 4}\right)+E\left(Y^{\prime 4}\right)+2 E\left(X^{\prime 2} Y^{\prime 2}\right)+\delta=\left\|X^{\prime 2}+Y^{\prime 2}\right\|_{2}^{2}+\delta .
\end{aligned}
$$

Together with Theorem 2.1 and Theorem 2.3 we obtain the following corollaries:

Corollary 3.4. Let $\left(X_{n}\right)$ be a sequence in $L^{4}(\mu)$ such that

(i) $\left\|X_{n+k}+X_{m+k}\right\|_{4}^{4} \leq\left\|X_{n}+X_{m}\right\|_{4}^{4}+\delta_{k}(m, n, k \in \mathbf{N})$,

(ii) $\left\|X_{n+k}-X_{m+k}\right\|_{4}^{4} \leq\left\|X_{n}-X_{m}\right\|_{4}^{4}+\delta_{k}(m, n, k \in \mathbf{N})$, with

(iii) $\lim _{k \rightarrow \infty} \delta_{k}=0$.

Then the sequence

$$
\frac{1}{n+1} \sum_{i=1}^{n} X_{i}^{2}
$$

is norm convergent in $L^{2}(\mu)$. 
Corollary 3.5. Let $K \subset L^{4}(\mu)$ and $T: K \longrightarrow K$ be such that

(i) $\|T f-T g\|_{4} \leq\|f-g\|_{4}(f, g \in K)$,

(ii) $\|T f+T g\|_{4} \leq\|f+g\|_{4}(f, g \in K)$.

Then for any $f \in K$

$$
\frac{1}{n+1} \sum_{i=0}^{n}\left(T^{i} f\right)^{2}
$$

is convergent in $L^{2}(\mu)$.

If moreover $K \subset L_{+}^{4}(\mu)$ then

$$
f \longrightarrow\left(T\left(f^{1 / 2}\right)\right)^{2}
$$

defines a mapping $\widetilde{T}$ of

$$
\widetilde{K}:=\left\{f^{2}: f \in K\right\}
$$

into itself satisfying

$$
\|\widetilde{T} f+\widetilde{T} g\|_{2} \leq\|f+g\|_{2} \quad(f, g \in \widetilde{K}) .
$$

Remark. The mapping $\widetilde{T}$ in Corollary 3.5 need not be nonexpansive, even if $T$ is a positive, linear $L^{1}-L^{\infty}$ contraction. To see this, let $(E, \mathscr{B}, \mu)$ be a probability space and define $T f=\int f d \mu I_{E}$. If there exists a measurable set $A$ with $\frac{1}{2} \leq \mu(A)<1$ then

$$
\left\|\widetilde{T} I_{E}-\widetilde{T} I_{A}\right\|_{2}=1-\mu(A)^{2} \geq \frac{3}{2}(1-\mu(A))>\sqrt{1-\mu(A)}=\left\|I_{E}-I_{A}\right\|_{2} .
$$

\section{ACKNOWLEDGMENTS}

The author would like to thank Professors Krengel and Lin for their helpful remarks and hints to the literature. I am also grateful to the referee for his very careful reading of the manuscript.

Note added in proof. For odd mappings Corollary 3.4 can already be found in J. B. Baillon, R. E. Bruck, S. Reich, On the asymptotic behavior of nonexpansive mappings and semigroups in Banach spaces, Houston J. Math. 4 (1978), 1-9. Of course, in this case 3.5(ii) follows from 3.5(i).

\section{REFERENCES}

1. J.-B. Baillon, Quelques propriétés de convergence asymptotique pour les contractions impaires, C. R. Acad. Sci. Paris 283 (1976), 587-590.

2. R. E. Bruck, Asymptotic hehavior of nonexpansive mappings, Contemp. Math. 18 (1983), 1-47.

3. B. Djafari-Rouhani and S. Kakutani, Ergodic theorems for nonexpansive nonlinear operators in a Hilbert space (unpublished preprint 1984).

4. N. Hirano and W. Takahashi, Nonlinear ergodic theorems for nonexpansive mappings in Hilbert spaces, Kodai Math. J. 2 (1975), 11-25. 
5. U. Krengel, Ergodic theorems, deGruyter, Berlin, New York, 1985.

6. U. Krengel and M. Lin, Order preserving nonexpansive operators in $L^{1}$, Israel J. Math. 58 (1987), 170-192.

Department of Mathematics, Princeton University, Fine Hall, Washington Road, Princeton, New Jersey 08544-1000 